\title{
Effects of Oral Vitamin B1 and Mecobalamin on Dry Eye Disease
}

\author{
Xiaotong Ren, ${ }^{1}$ Yilin Chou, ${ }^{1}$ Xiaodan Jiang, ${ }^{1}$ Ran Hao,, ${ }^{1}$ Yuexin Wang, ${ }^{1}$ Yanyan Chen, \\ and Xuemin $\mathrm{Li} \mathbb{D D}^{1}$ \\ ${ }^{1}$ Department of Ophthalmology, Peking University Third Hospital, Beijing, China \\ ${ }^{2}$ Department of Ophthalmology, Daqing Oilfield General Hospital, Daqing, Heilongjiang, China
}

Correspondence should be addressed to Xuemin Li; lxmlxm66@sina.com

Received 18 May 2020; Accepted 28 August 2020; Published 9 September 2020

Academic Editor: Katarzyna Krysik

Copyright ( 2020 Xiaotong Ren et al. This is an open access article distributed under the Creative Commons Attribution License, which permits unrestricted use, distribution, and reproduction in any medium, provided the original work is properly cited.

Purpose. To assess the effects of oral vitamin B1 and mecobalamin on dry eye disease (DED) and patient satisfaction with treatment. Methods. In this randomized controlled study, DED patients were divided into 4 groups based on treatment: group 1, only artificial tears; group 2, corticosteroid eye drops and artificial tears; group 3, oral vitamin B1, mecobalamin, and artificial tears; and group 4, oral vitamin B1, mecobalamin, corticosteroid eye drops, and artificial tears. DED symptoms, signs, and patient satisfaction with treatment were assessed at baseline and at 1 and 2 months after treatment. Results. In total, 152 eyes from 76 patients (age, $55.25 \pm 18.16$ years) were included. In group 3 , there were significant differences in dryness, foreign body sensation, burning, and tear film breakup time first (TBUTF) between 1 and 2 months after treatment and in satisfaction scores before and after treatment $(P<0.05)$. In group 3 , there were also significant differences in dryness, foreign body sensation, photophobia, and TBUTA and between baseline and 2 months after treatment $(P<0.05)$. There was a significant difference in foreign body sensation between 1 and 2 months after treatment in groups 3 and $4(P<0.05)$. Furthermore, we also find obvious improvement in corneal nerve fiber density (CNFD) between baseline and 1 and 2 months after treatment in groups 3 and $4(P<0.05)$. Conclusions. Oral vitamin $\mathrm{B} 1$ and mecobalamin can relieve some dry eye symptoms such as dryness, pain, and photophobia and improve DED signs and patient satisfaction. Thus, vitamin B1 and mecobalamin are potential treatment options for patients with DED.

\section{Introduction}

Dry eye is a multifactorial disease of the ocular surface characterized by loss of tear film homeostasis and ocular symptoms. Instability of the tear film, inflammation and injury of the ocular surface, and neurosensory abnormalities all play important roles in dry eye [1]. Pain related to dry eye disease (DED) is transmitted through the peripheral axons of trigeminal ganglion (TG) neurons innervating the cornea and conjunctiva [2]. The cornea is one of the densest nervous tissues [2] and is responsible for various sensations in the eye, such as pain, touch, and temperature. The trigeminal nerve also plays an essential role in the corneal reflex and tear production [3]. DED can cause damage to corneal epithelial cells, including keratosis and exfoliation, which are related to acute axonal injury during corneal nerve damage [4] and lead to symptoms such as pain and photophobia [5]. In vivo confocal microscopy (IVCM) examinations of DED patients have also revealed loss of parallelization and disordered arrangement in the corneal subepithelial nerve $[5,6]$.

Vitamin B1 (thiamine) given perineurally exerts analgesic effects through axonal flow by enhancing the synthesis of acetylcholine in dorsal horn inhibitory neurons $[7,8]$. In addition, vitamin B12 can repair peripheral nerves by inducing the proliferation of Schwann cells and regeneration of myelinated nerve fibers. Cobalamin is the endogenous form of vitamin B12 and is superior to other forms of vitamin B12, such as cyanocobalamin and cobalamin adenosine, for the treatment of peripheral neuropathy $[9,10]$. However, the effects of oral vitamin B1 and cobalamin on DED have not been investigated. Some studies have shown that local application of vitamin B12 is effective for corneal recovery and reduces corneal neuropathic pain [11]. Nevertheless, it may be insufficient to evaluate the efficacy of DED treatment using only objective clinical indicators, as 
patient satisfaction is also extremely important in the treatment process $[12,13]$. Therefore, the purpose of this study was to investigate the effects of oral vitamin B1 and cobalamin on signs and symptoms of dry eye and treatment satisfaction in patients with DED in order to help tailor the treatment strategy to patient needs.

\section{Methods}

The study was approved by the Human Research and Ethics Committee of Peking University Third Hospital (No. M2018149), adhered to the tenets of the Declaration of Helsinki, and was performed at Peking University Third Hospital Eye Center from October 2018 to March 2019. Written informed consent was obtained from each patient before enrollment in the study.

2.1. Participants. A randomized controlled trial was conducted at Peking University Third Hospital. DED was diagnosed according to the diagnostic criteria established by the Tear Film and Ocular Surface Society (TFOS) Dry Eye WorkShop II (DEWS II) [14]. Inclusion criteria included positive symptoms (Dry Eye Questionnaire-5 $>5$ or ocular surface disease index $(\mathrm{OSDI})>13$ ) and at least one of the following signs: noninvasive breakup time (BUT) $<10 \mathrm{sec}-$ onds; osmolarity $>308 \mathrm{mOsm} / \mathrm{L}$ and interocular difference $>8 \mathrm{mOsm} / \mathrm{L}$; and corneal staining $>5$ spots and conjunctival staining $>9$ spots. Patients who had other corneal diseases, glaucoma, macular degeneration, optic neuropathy, uveitis, diabetic retinopathy, or complex systemic disease or were incapable of carrying out study-related visits were excluded.

2.2. Experimental Design. DED patients with corneal nerve layer injury were divided into the Langerhans group and non-Langerhans group based on the presence of Langerhans cells in the corneal nerve layer on IVCM. Patients in the former group were administered with corticosteroid eye drops (Flumetholon, Saten, Japan) and randomly assigned to receive oral vitamin B1 (vitamin B1 tablets, Shanxi Hengruida Pharmaceutical Co., China), mecobalamin (mecobalamin tablets, Weicai (China) Pharmaceutical Co., China), and/or artificial tears (Hycosan, URSAPHARM, Germany, and Systane Ultra Lubricant Eye Drops, Alcon Laboratories, USA). Patients in the latter group were randomly assigned to receive oral vitamin $\mathrm{B} 1$, mecobalamin, and/or artificial tears (Hycosan, URSAPHARM, Germany, and Systane Ultra Lubricant Eye Drops, Alcon Laboratories, USA) or artificial tears alone as a control. Thus, the patients were ultimately divided into 4 groups: group 1, only artificial tears; group 2, corticosteroid eye drops and artificial tears; group 3, oral vitamin B1, mecobalamin, and artificial tears; and group 4, oral vitamin B1, mecobalamin, corticosteroid eye drops, and artificial tears. Each patient underwent continuous treatment for 2 months and was subjected to clinical assessment before treatment (baseline) and at 1 and 2 months after treatment. The treatments and follow-up protocols for all groups are shown in Figure 1.
2.3. Evaluation Index. At every follow-up, each participant completed a questionnaire regarding subjective symptoms and satisfaction with treatment [15]. Slit lamp examination and other objective and auxiliary screenings were conducted to evaluate signs of DED. Each examination was carried out in the same examination room under the same conditions by a single doctor.

A Placido ring-based, noncontact corneal topographer (Keratograph $5 \mathrm{M}$; OCULUS, Wetzlar, Germany) was used to evaluate the tear film [16], including the tear meniscus height (TMH), tear film breakup time first (TBUTF), and tear film breakup time average (TBUTA). In order to ensure a dark background in corneal topography, automatic red-ring illumination was used. An illuminated ring pattern was projected onto the cornea using the corneal topographer, which features a 22-ring Placido disk on which four infrared diodes arranged in two parallel rows are fixed horizontally. To avoid errors, the examination was repeated three times. During each examination, patients were asked to keep their eyes open for as long as possible after 3 to 4 blinks. From this point on, the examiner began to observe the corneal reflex image. When tear film breakup occurred or became unstable, the reflection image became irregular, and the tear film breakup time was obtained. The first value was the TBUTF, and the mean of three values was the TBUTA. The distance between the light band at the junction of the corneal conjunctival surface and the lower eyelid margin was the TMH.

IVCM was used to observe the microstructure of the cornea. All patients were examined with a digital corneal confocal laser-scanning microscope (HRT II RCM Heidelberg Engineering Inc., Heidelberg, Germany, Rostock Cornea Module) equipped with the built-in software Heidelberg Eye Explorer version 1.5.10.0. Two-dimensional images with a definition of $384 * 384$ pixels over an area of $400 \mu \mathrm{m} * 400 \mu \mathrm{m}$, lateral spatial resolution of $0.5 \mu \mathrm{m}$, and depth resolution of $1-2 \mu \mathrm{m}$ were captured. For each eye, 30-40 images were acquired from the corneal epithelium to the endothelium. Five images of good quality were chosen to quantify corneal nerves using a pretrained deep learning model [17], and the average corneal nerve fiber density (CNFD) was recorded.

All patients were asked to complete two questionnaires to evaluate their symptoms and satisfaction with treatment before and at 1 month and 2 months after treatment. The patients responded to the questions using a 10-point scale. The first questionnaire comprised 10 questions (questions 1-10) related to $10 \mathrm{DED}$ symptoms, including dryness, foreign body sensation, pain, burning, watering, asthenopia, blurred vision, itching, increased secretions, and photophobia. The responses ranged from "have no feelings (score $0)$ " to "very serious (score 10)." The second questionnaire was related to satisfaction with treatment and included 4 questions regarding how the patients felt about their condition before and after treatment (responses ranged from "very bad (score 0)" to "very good (score 10)"), the ease of following doctors' advice (from "very difficult (score 0)" to "very easy (score 10)"), and improvement after treatment (from "none (score 0)" to "significantly improved (score 10)”). 


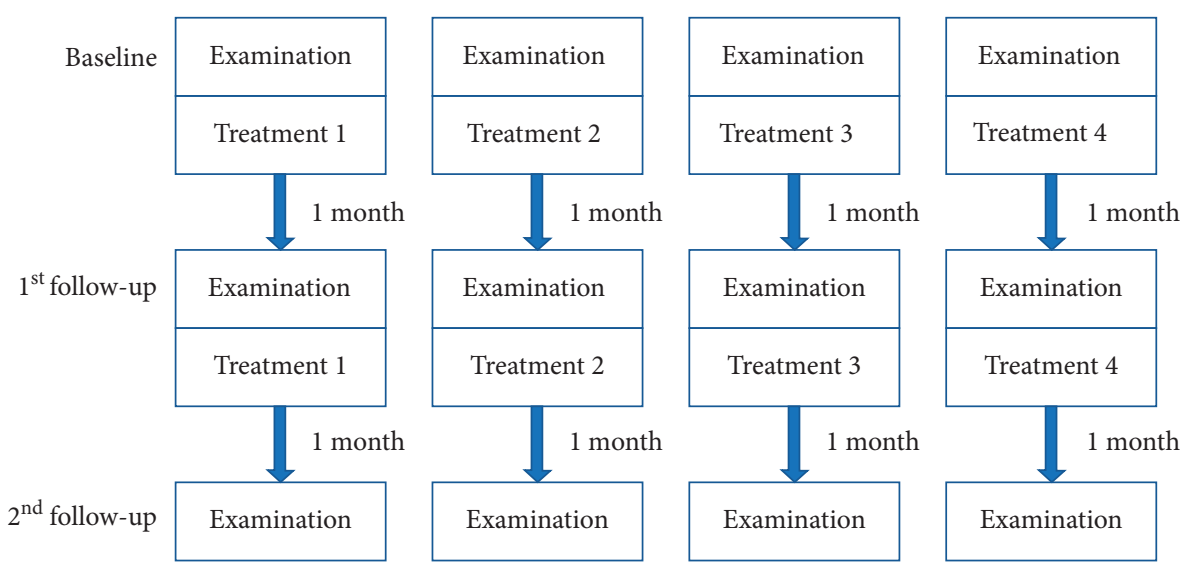

FIGURE 1: Treatment 1 (group 1): only artificial tears; treatment 2 (group 2): corticosteroids and artificial tears; treatment 3 (group 3): oral vitamin B1, mecobalamin, and artificial tears; treatment 4 (group 4): oral vitamin B1, mecobalamin, corticosteroids, and artificial tears.

TABLE 1: Basic information.

\begin{tabular}{lcccc}
\hline & Group 1 & Group 2 & Group 3 & Group 4 \\
\hline Sex (male \%) & 20 & 30 & 25 & 31.25 \\
Age (years, mean \pm SD) & $57.35 \pm 16.37$ & $51.90 \pm 18.57$ & $53.85 \pm 17.93$ & $58.56 \pm 19.40$ \\
Number & 40 & 40 & 40 & ns \\
\hline
\end{tabular}

ns: no statistical significance.

The questionnaire scores, satisfaction scores, TMH, TBUTF, TBUTA, and CNFD of each group at each time point were compared within the individual groups and between groups to evaluate the therapeutic effects of the different treatments.

2.4. Statistical Analysis. SPSS software version 22 (SPSS Inc., Chicago, IL, USA) was used for statistical analysis. The Kolmogorov-Smirnov test was used to check the normality of the data distribution. Descriptive data are presented as the mean and standard deviation (SD). The mixed linear model was used to compare differences before and after treatment. To analyze repeated measurements, the patient was selected as the subject, and the time point was set as the repeated factor. In the model, the covariance type was chosen based on the covariance matrix from our preliminary analysis. $P$ values less than 0.05 were considered statistically significant.

\section{Results}

3.1. Patient Characteristics. A total of 152 eyes from 76 individuals (age, $55.25 \pm 18.16$ years; 56 females) were included and divided into 4 groups according to treatment. Significant age and sex differences were not found among the groups. The demographic characteristics of the patients are shown in Table 1.

3.2. Comparison of Pre- and Posttreatment Signs, Symptoms, and Treatment Satisfaction Scores in Each Group. As shown in Table 2, in group 3, dryness, foreign body sensation, burning, and TBUTF differed significantly between 1 and 2 months after treatment, and satisfaction scores differed significantly before and after treatment $(P<0.05)$. In group 3 , there were also significant differences in dryness, foreign body sensation, and photophobia and TBUTA between baseline and 2 months after treatment $(P<0.05)$. In group 4 , pain, blurred vision, and the total symptom score were significantly different between baseline and 1 month after treatment $(P<0.05)$, and asthenopia differed significantly between 1 and 2 months after treatment (3.97 \pm 3.34 , $5.23 \pm 3.19, P<0.05)$. Interestingly, in both groups 3 and 4 , CNFD increased at 1 and 2 months after treatment $(P<0.05)$. However, in group 4 , the Langerhans cells in the corneal nerve layer were obviously reduced at 1 month after treatment, as shown in Figure 2. Finally, in group 1, significant differences were observed in dryness between baseline and 1 month after treatment and in blurred vision between 1 and 2 months after treatment and between baseline and 2 months after treatment $(P<0.05)$.

3.3. Comparison of Pre- and Posttreatment Signs, Symptoms, and Treatment Satisfaction Scores among Groups. As shown in Table 3, the changes in dryness between baseline and 1 and 2 months after treatment differed significantly between groups 1 and $4(P<0.05)$, and the changes in dryness between 1 and 2 months after treatment differed significantly between groups $2 / 4$ and $3(P<0.05)$. The changes in foreign body sensation between 1 and 2 months after treatment differed significantly between groups $2 / 3$ and $4(P<0.05)$, and the changes in photophobia between 1 and 2 months after treatment differed significantly between groups $2 / 3$ and $4(P<0.05)$. The changes in the total symptom score between baseline and 1 and 2 months after treatment differed significantly between groups $1 / 2$ and $4(P<0.05)$. In addition, 


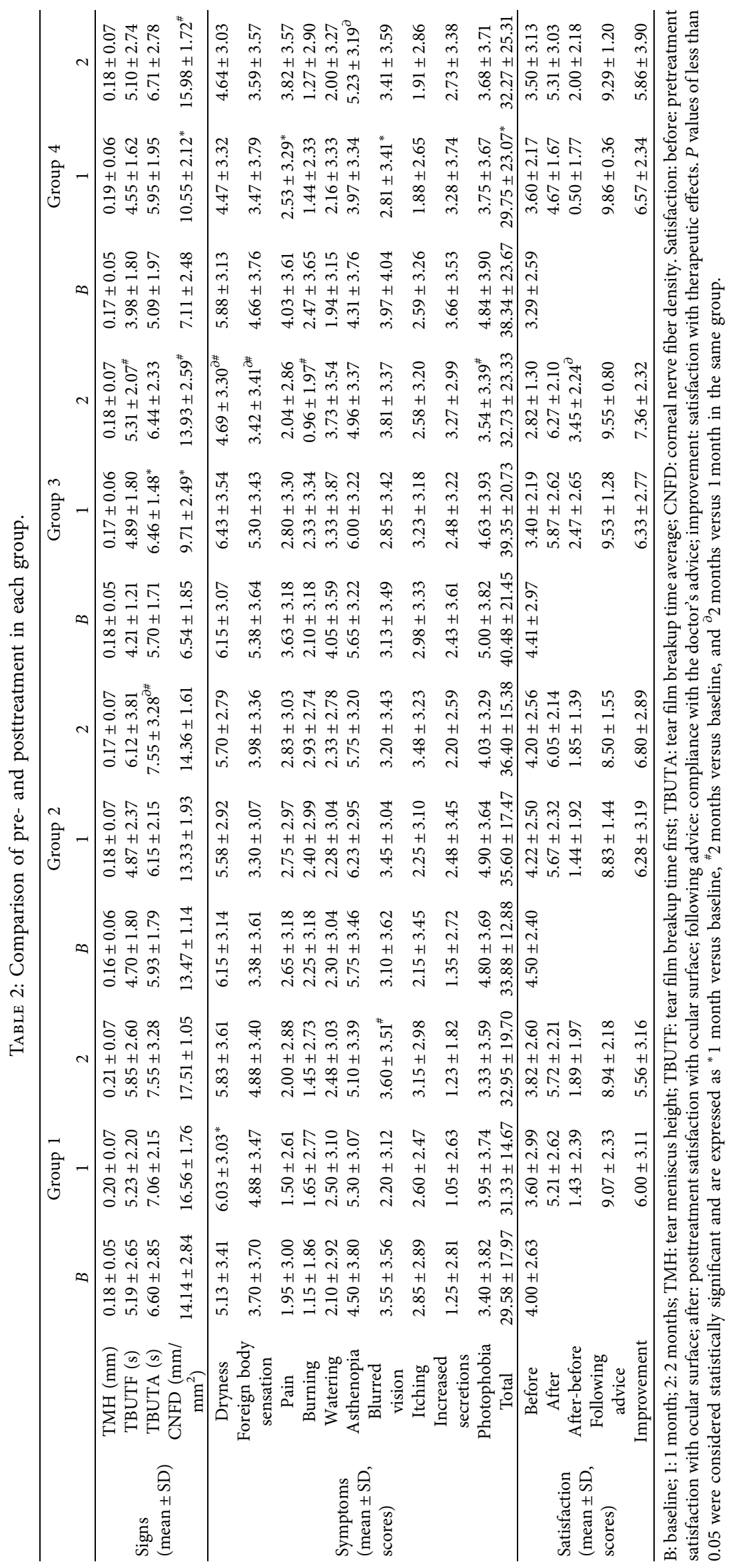




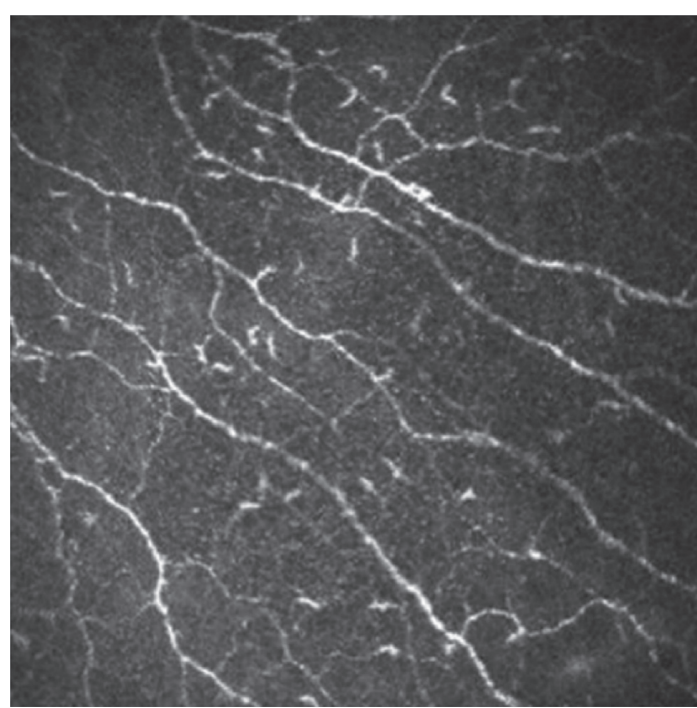

(a)

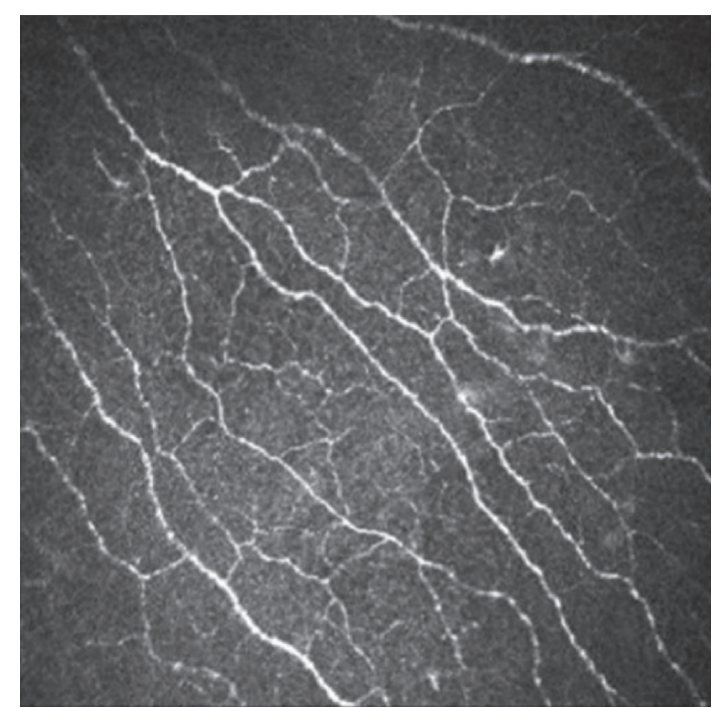

(b)

Figure 2: These two images are from the same corneal nerve layer of subepithelial corneal epithelium of the IVCM in the same patient in group 4: (a) before treatment; (b) 1 month after treatment.

the change in CNFD between baseline and 1 and 2 months after treatment differed significantly between groups 2 and $3 / 4(P<0.05)$, while the change between 1 and 2 months after treatment differed significantly between groups $1 / 2$ and $3(P<0.05)$.

\subsection{Correlations between Pre- and Posttreatment Signs/} Symptoms/Treatment Satisfaction Scores and Sex/Age. The correlations between pre- and posttreatment signs/symptoms/treatment satisfaction scores and sex/age are shown in Table 4. In group 3, dryness and photophobia improved to a significantly greater extent in men, while the improvements in TBUTF and itching were significantly greater in women. TBUTF, TBUTA, burning, itching, and the total symptom score improved to a significantly greater extent in the older age group compared with the younger age group.

\section{Discussion}

DED is a multifactorial disease with various symptoms and signs [1]. Many treatments for dry eye improve only some symptoms/signs, while other symptoms, particularly pain and photophobia, are neglected. Therefore, there is a need for new convenient treatment measures. To provide new ideas for diagnosis and treatment, this study evaluated whether oral vitamin B1 and mecobalamin can improve the signs/symptoms/satisfaction of patients with DED.

In this study, the scores of four dry eye symptoms (dryness, foreign body sensation, burning, and photophobia) and TBUTF improved significantly at 2 months after treatment with oral vitamin B1, mecobalamin, and artificial tears (group 3). When corticosteroid eye drops were added to this treatment (group 4), pain, blurred vision, and total symptom scores were significantly improved at 1 month after treatment. We also observed an obvious decrease in
Langerhans cells in the corneal nerve layer at 1 month after treatment. In addition, the CNFD proved obviously improved in both groups 3 and 4 at 1 and 2 months after treatment. These observations suggest that oral vitamin B1 and mecobalamin can help nourish and repair the corneal nerve layer to some extent, thereby alleviating burning and photophobia.

The cornea possesses approximately a hundred times more nociceptors than the dermis. These nociceptors are located slightly below the lacrimal layer, between the epithelial cells, and are unmyelinated, leading to extreme sensitivity in nociceptive sensation $[18,19]$. The severity of dry eye may depend on the degree of changes in the structure and function of the subcorneal nerve [20]; greater severity of dry eye is associated with a larger degree of changes in nerve branches and curvature on IVCM [21]. Ocular surface neuropathy can induce or aggravate dry eye, and long-term serious dry eye can produce changes in the structure and function of ocular surface nerves, resulting in a vicious circle $[5,21]$. Corneal nerve injury can lead to acute axonal injury, which can reduce the threshold potential of ion channels in corneal nerve endings due to the release of inflammatory mediators such as substance $P$, tumor necrosis factor- $\alpha$, and interleukin-1 [4]. The simultaneous release of nerve growth factor during this process leads to gene expression and upregulation, resulting in an excessive corneal nociceptor response [4], which might account for symptoms of pain, burning, and photophobia. By contrast, repeated or severe axonal injury can shift the corneal nociceptor's functional state to nerve regeneration rather than signal transduction $[18,22]$, temporarily alleviating symptoms. During regeneration, the damaged nerves can form nerve bundles and microneuromas, which may also cause spontaneous pain, burning, and photophobia [18].

Previous studies have shown that the analgesic effect of vitamin B1 occurs via indirect enhancement of axonal flow 


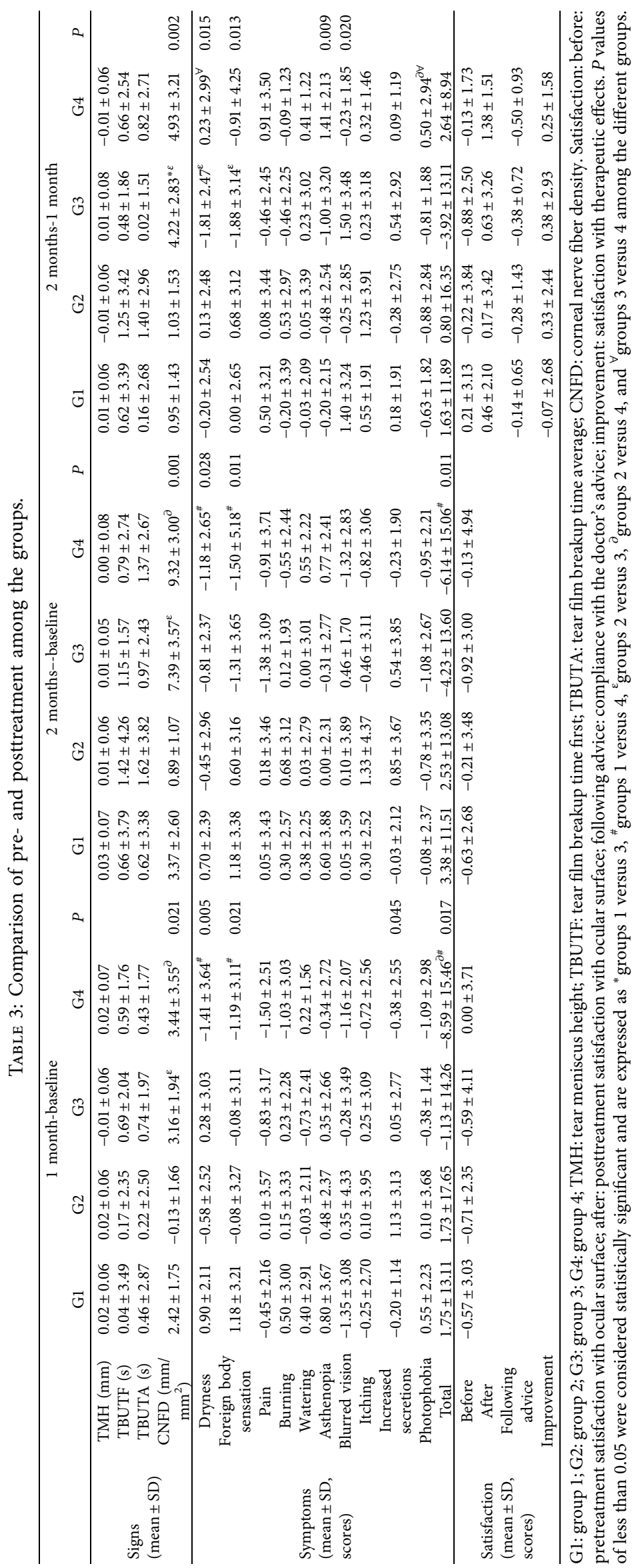


TABLE 4: The correlations between pre- and posttreatment signs/symptoms/treatment satisfaction scores and sex/age.

\begin{tabular}{|c|c|c|c|c|c|c|c|c|c|}
\hline & & \multicolumn{2}{|c|}{ G1 } & \multicolumn{2}{|c|}{$\mathrm{G} 2$} & \multicolumn{2}{|c|}{ G3 } & \multicolumn{2}{|c|}{$\mathrm{G} 4$} \\
\hline & & $\begin{array}{l}\text { Estimate } \\
\quad(\mathrm{sex})\end{array}$ & $\begin{array}{l}\text { Estimate } \\
\text { (age) }\end{array}$ & $\begin{array}{l}\text { Estimate } \\
\quad(\mathrm{sex})\end{array}$ & $\begin{array}{l}\text { Estimate } \\
\text { (age) }\end{array}$ & $\begin{array}{l}\text { Estimate } \\
\quad(\mathrm{sex})\end{array}$ & $\begin{array}{l}\text { Estimate } \\
\text { (age) }\end{array}$ & $\begin{array}{l}\text { Estimate } \\
\quad(\mathrm{sex})\end{array}$ & $\begin{array}{c}\text { Estimate } \\
\text { (age) }\end{array}$ \\
\hline \multirow{3}{*}{ Signs } & TMH & Ns & $\mathrm{ns}$ & 0.056 & ns & ns & $\mathrm{ns}$ & $\mathrm{ns}$ & ns \\
\hline & TBUTF & Ns & ns & ns & -0.029 & 0.719 & -0.025 & ns & ns \\
\hline & TBUTA & ns & $\mathrm{ns}$ & $\mathrm{ns}$ & ns & ns & -0.034 & ns & $\mathrm{ns}$ \\
\hline \multirow{9}{*}{ Symptoms } & Dryness & ns & $\mathrm{ns}$ & ns & -0.065 & -1.892 & ns & -2.182 & ns \\
\hline & Pain & ns & -0.060 & ns & -0.891 & ns & ns & ns & -0.083 \\
\hline & Burning & ns & $\mathrm{ns}$ & ns & ns & ns & -0.048 & -2.072 & ns \\
\hline & Watering & ns & ns & ns & ns & $\mathrm{ns}$ & ns & -2.734 & ns \\
\hline & Asthenopia & ns & ns & ns & -0.085 & ns & ns & ns & -0.054 \\
\hline & Blurred vision & ns & ns & 1.820 & ns & ns & ns & ns & 0.069 \\
\hline & Itching & ns & ns & ns & $\mathrm{ns}$ & 2.012 & -0.091 & -2.636 & ns \\
\hline & Photophobia & ns & ns & 2.736 & ns & -2.969 & ns & -3.873 & 0.054 \\
\hline & Total & $\mathrm{ns}$ & $\mathrm{ns}$ & ns & ns & ns & -0.346 & -26.519 & ns \\
\hline \multirow{5}{*}{ Satisfaction } & Before & ns & ns & ns & ns & ns & 0.030 & -1.579 & -0.113 \\
\hline & After & ns & ns & ns & ns & ns & -0.049 & 3.627 & -0.055 \\
\hline & After-before & ns & ns & ns & ns & ns & -0.082 & ns & ns \\
\hline & $\begin{array}{c}\text { Following } \\
\text { advice }\end{array}$ & -2.223 & ns & 0.951 & ns & $\mathrm{ns}$ & ns & -0.019 & 0.003 \\
\hline & Improvement & $\mathrm{ns}$ & $\mathrm{ns}$ & $\mathrm{ns}$ & ns & ns & ns & ns & -0.136 \\
\hline
\end{tabular}

G1: group 1; G2: group 2; G3: group 3; G4: group 4; TMH: tear meniscus height; TBUTF: tear film breakup time first; TBUTA: tear film breakup time average; Satisfaction: before: pretreatment satisfaction with ocular surface; after: posttreatment satisfaction with ocular surface; following advice: compliance with the doctor's advice; improvement: satisfaction with therapeutic effects. ns: no statistical significance.

$[7,8,23]$. We believe that, in the corneal nerve, this effect of vitamin B1 can lead to relief of pain. Cobalamin, an endogenous form of vitamin B12, is present in the blood and medullary fluid. Compared with other forms of vitamin B12, cobalamin has superior effects on neuronal transmission. Cobalamin can promote nucleic acid, protein, and lipid metabolism through methyl conversion reactions [10]. As a coenzyme, cobalamin participates in the synthesis of thymine from deoxynucleoside, promotes the metabolism of nucleic acid protein through methyl transformation reactions, serves as a cofactor in the conversion of homocysteine to methionine, and promotes the formation of the axonal myelin sheath and axonal transport, thus repairing damaged nerve tissue [9]. Vitamin B12 is widely used in the treatment of pain, including diabetic neuropathy, herpes, and surgeryrelated pain. Some researchers have reported that patients with vitamin B12 deficiency and ocular neuropathic pain respond effectively to vitamin B12 treatment and are able to discontinue all topical medications within a few weeks of treatment [24]. By contrast, a period of two months was required to observe effectiveness in the treatment group in the present study. This discrepancy may be related to the mode of administration and interactions with other drugs. Some in vivo studies have shown that the analgesic effect of vitamin B12 is due to the activation of opioid receptors or increased serotonin levels in different regions of the brain, which enhance the inhibitory effect of afferent noxious neurons in the spinal cord and, in turn, lead to weakening of the thalamic neuronal response to noxious stimuli. In addition, vitamin B12 eye drops have been reported to alleviate dry eye symptoms by reducing oxidative stress and inflammation [25]. However, there are no reliable studies of its pharmacokinetics, and hence we cannot conclude whether the effectiveness of local instillation differs from that of oral administration. Vitamin B12 has also been reported to improve corneal reinnervation and reepithelization after injury [26], and the changes in CNFD observed in the present study partially confirm these findings.

The significant differences in satisfaction scores between baseline and 1 and 2 months after treatment in group 3 are consistent with previous reports and are not unexpected because photophobia, burning, and pain are subjective symptoms and have a greater impact on satisfaction scores $[12,27]$. Corticosteroid supplementation has also been shown to be helpful in reducing ocular surface inflammation, as corticosteroids inhibit proinflammatory cytokines [28]. Corticosteroids exert a powerful anti-inflammatory effect by acting on subbasal dendritic cells in the corneal nerve layer and activated Langerhans cells, both of which play roles in inflammation $[29,30]$. This explains the beneficial effects of corticosteroids on group 4, which included significant improvements in pain, blurred vision, and total symptom scores and were similar to the results of earlier studies [29]. The significant reduction in Langerhans cells in the corneal nerve layer in group 4 at 1 month after treatment partially supports this conjecture. However, similar changes were not observed in group 2, in which both corticosteroid eye drops and artificial tears were given, suggesting a synergistic effect of corticosteroids, vitamin B1, and mecobalamin. However, withdrawal of the corticosteroid after 1 month may have led to rebound of some symptoms, which has rarely been reported in previous studies.

Unexpectedly, demographic differences in the effectiveness of the treatments for DED were observed: oral vitamin $\mathrm{B} 1$ and mecobalamin were more effective for men than women, particularly for dryness and photophobia. We 
speculate that this may be related to the decline in hormone levels in older women, but additional experiments are needed to confirm this possibility. These findings could be used to guide treatment in the future.

In summary, our study showed that oral vitamin B1 and mecobalamin can relieve some DED symptoms such as dryness, foreign body sensation, and photophobia; stabilize the tear film; and improve patient satisfaction with regard to treatment. We speculate that neurotrophism may be one of the mechanisms underlying these effects. However, at least 2 months of treatment were required for these benefits to be evident, which demands patient compliance. Corticosteroid eye drops can help relieve the overall symptoms of dry eyes, but any withdrawal may cause rebound.

Our study provides new therapeutic options for the treatment of DED as well as guidance regarding the duration and combined use of different DED treatments. A limitation of this study is the relatively small sample size. In addition, this was a trial treatment, and we did not check serum vitamin B1 and vitamin B12 levels in the DED patients. We do not know whether there is synergy or interaction between vitamin $\mathrm{B} 1$ and mecobalamin, or do we know which is more effective in the treatment of DED. Answering these questions will be the focus of our subsequent research. As noted previously $[11,30]$, continuous patient education and counseling should be combined with pharmaceutical treatment for the management of complex diseases such as DED.

\section{Abbreviations}

BUT: $\quad$ Breakup time

DED: Dry eye disease

TBUTA: Tear film breakup time average

TBUTF: Tear film breakup time first

TMH: $\quad$ Tear meniscus height

IVCM: In vivo confocal microscopy

CNFD: Corneal nerve fiber density.

\section{Data Availability}

The data used to support the findings of this study are available from the first author upon request.

\section{Disclosure}

An earlier version of the manuscript has been presented as a conference abstract in The 24th Congress of Chinese Ophthalmological Society. The sponsor or funding organization had no role in the design or conduct of this research.

\section{Conflicts of Interest}

All authors declare that they have no conflicts of interest.

\section{Authors' Contributions}

Xiaotong Ren, Yilin Chou, Xiaodan Jiang, and Ran Hao contributed equally to this work.

\section{Acknowledgments}

The publication of this paper was supported by a grant from Capital's Funds for Health Improvement and Research (Grant no. CFH2018-2-4093).

\section{References}

[1] J. P. Craig, K. K. Nichols, E. K. Akpek et al., "TFOS DEWS II definition and classification report," The Ocular Surface, vol. 15, no. 3, pp. 276-283, 2017.

[2] C. Belmonte, J. J. Nichols, S. M. Cox et al., "TFOS DEWS II pain and sensation report," The Ocular Surface, vol. 15, no. 3, pp. 404-437, 2017.

[3] B. S. Shaheen, M. Bakir, and S. Jain, "Corneal nerves in health and disease," Survey of Ophthalmology, vol. 59, no. 3, pp. 263-285, 2014.

[4] S. Goyal and P. Hamrah, "Understanding neuropathic corneal pain-gaps and current therapeutic approaches," Seminars in Ophthalmology, vol. 31, no. 1-2, pp. 59-70, 2016.

[5] A. Kheirkhah, T. H. Dohlman, F. Amparo et al., "Effects of corneal nerve density on the response to treatment in dry eye disease," Ophthalmology, vol. 122, no. 4, pp. 662-668, 2015.

[6] G. Giannaccare, M. Pellegrini, S. Sebastiani, F. Moscardelli, P. Versura, and E. C. Campos, "In vivo confocal microscopy morphometric analysis of corneal subbasal nerve plexus in dry eye disease using newly developed fully automated system," Graefe's Archive for Clinical and Experimental Ophthalmology, vol. 257, no. 3, pp. 583-589, 2019.

[7] F. Alemanno, D. Ghisi, B. Westermann et al., "The use of vitamin $\mathrm{B} 1$ as a perineural adjuvant to middle interscalene block for postoperative analgesia after shoulder surgery," Acta Bio-Medica: Atenei Parmensis, vol. 87, no. 1, pp. 22-27, 2016.

[8] G. Xû, G. Xú, Y. Feng, W. Z. Tang, and Z. W. Lv, "Transcutaneous electrical nerve stimulation in combination with cobalamin injection for postherpetic neuralgia," American Journal of Physical Medicine \& Rehabilitation, vol. 93, no. 4, pp. 287-298, 2014.

[9] C. Zheng, W. Ou, H. Shen et al., "Combined therapy of diabetic peripheral neuropathy with breviscapine and mecobalamin: a systematic review and a meta-analysis of Chinese studies," BioMed Research International, vol. 2015, Article ID 680756, 11 pages, 2015.

[10] L. L. Zhang, Y. H. Wang, Z. H. Shao et al., "Prophylaxis of bortezomib-induced peripheral neuropathy in patients with multiple myeloma by high-dose intravenous mecobalamin," Zhongguo Shi Yan Xue Ye Xue Za Zhi, vol. 25, no. 2, pp. 480-484, 2017.

[11] S. B. Han, H. K. Yang, J. Y. Hyon, and W. R. Wee, "Association of dry eye disease with psychiatric or neurological disorders in elderly patients," Clinical Interventions in Aging, vol. 12, pp. 785-792, 2017.

[12] J. A. P. Gomes and R. M. Santo, "The impact of dry eye disease treatment on patient satisfaction and quality of life: a review," The Ocular Surface, vol. 17, no. 1, pp. 9-19, 2019.

[13] A. Crnej, A. Kheirkhah, A. Ren et al., "Patients' perspectives on their dry eye disease," The Ocular Surface, vol. 14, no. 4, pp. 440-446, 2016.

[14] J. S. Wolffsohn, R. Arita, R. A. Chalmers et al., "TFOS DEWS II diagnostic methodology report," The Ocular Surface, vol. 15, no. 3, pp. 539-574, 2017.

[15] X. Djalilian, H. Lv, H. Song et al., "Evaluation of the safety and effectiveness of intense pulsed light in the treatment of 
meibomian gland dysfunction," Journal of Ophthalmology, vol. 2016, Article ID 1910694, 8 pages, 2016.

[16] J. Hong, X. Sun, A. Wei et al., "Assessment of tear film stability in dry eye with a newly developed keratograph," Cornea, vol. 32, no. 5, pp. 716-721, 2013.

[17] S. Wei, X. Ren, Y. Wang et al., "Therapeutic effect of intense pulsed light (IPL) combined with meibomian gland expression (MGX) on meibomian gland dysfunction (MGD)," Journal of Ophthalmology, vol. 2020, Article ID 3684963, 7 pages, 2020.

[18] P. Rosenthal, I. Baran, and D. S. Jacobs, "Corneal pain without stain: is it real?” The Ocular Surface, vol. 7, no. 1, pp. 28-40, 2009.

[19] L. J. Müller, C. F. Marfurt, F. Kruse, and T. M. T. Tervo, "Corneal nerves: structure, contents and function," Experimental Eye Research, vol. 76, no. 5, pp. 521-542, 2003.

[20] A. Labbé, Q. Liang, Z. Wang et al., "Corneal nerve structure and function in patients with non-sjögren dry eye: clinical correlations," Investigative Opthalmology \& Visual Science, vol. 54, no. 8, pp. 5144-5150, 2013.

[21] O. Tanriverdi, M. D. Aydin, M. R. Onen et al., "Understanding of dry eye in subarachnoid hemorrhage: an experimental study on the role of facial nerve ischemia," Turkish Neurosurgery, vol. 29, no. 3, pp. 362-368, 2019.

[22] P. Rosenthal and D. Borsook, "The corneal pain system. Part I: the missing piece of the dry eye puzzle," The Ocular Surface, vol. 10, no. 2, pp. 2-14, 2012.

[23] L. Negrão, P. Almeida, S. Alcino et al., "Effect of the combination of uridine nucleotides, folic acid and vitamin B12 on the clinical expression of peripheral neuropathies," Pain Management, vol. 4, no. 3, pp. 191-196, 2014.

[24] R. Shetty, K. Deshpande, A. Ghosh, and S. Sethu, "Management of ocular neuropathic pain with vitamin B12 supplements," Cornea, vol. 34, no. 10, pp. 1324-1325, 2015.

[25] A. Macri, C. Scanarotti, A. M. Bassi et al., "Evaluation of oxidative stress levels in the conjunctival epithelium of patients with or without dry eye, and dry eye patients treated with preservative-free hyaluronic acid $0.15 \%$ and vitamin B12 eye drops," Graef's archive for Clinical and Experimental Ophthalmology = Albrecht von Graefes Archiv Fur Klinische und Experimentelle Ophthalmologie, vol. 253, no. 3, pp. 425-430, 2015.

[26] M. R. Romano, F. Biagioni, A. Carrizzo et al., "Effects of vitamin $\mathrm{B} 12$ on the corneal nerve regeneration in rats," $E x$ perimental Eye Research, vol. 120, pp. 109-117, 2014.

[27] T. Deveney and P. A. Asbell, "Patient and physician perspectives on the use of cyclosporine ophthalmic emulsion $0.05 \%$ for the management of chronic dry eye," Clinical Ophthalmology (Auckland, N.Z.), vol. 12, pp. 569-576, 2018.

[28] E. Villani, E. Garoli, V. Termine, F. Pichi, R. Ratiglia, and P. Nucci, "Corneal confocal microscopy in dry eye treated with corticosteroids," Optometry and Vision Science, vol. 92, no. 9, pp. e290-295, 2015.

[29] "Management and therapy of dry eye disease: report of the management and therapy subcommittee of the international dry eye WorkShop (2007)," The Ocular Surface, vol. 5, no. 2, pp. 163-178, 2007.

[30] E. J. Holland, M. Darvish, K. K. Nichol et al., "Efficacy of topical ophthalmic drugs in the treatment of dry eye disease: a systematic literature review," The Ocular Surface, vol. 17, no. 3, pp. 412-423, 2019. 